Article

\title{
The Application of Impedance Spectroscopy for Pseudomonas Biofilm Monitoring during Phage Infection
}

\author{
Grzegorz Guła ${ }^{1}$, Paulina Szymanowska ${ }^{2}{ }^{-}$, Tomasz Piasecki ${ }^{2}$, Sylwia Góras ${ }^{1}$, \\ Teodor Gotszalk ${ }^{2}$ and Zuzanna Drulis-Kawa ${ }^{1, *(D)}$ \\ 1 Department of Pathogen Biology and Immunology, Institute of Genetics and Microbiology, \\ University of Wroclaw, 51-148 Wroclaw, Poland; grzegorz.gula@uwr.edu.pl (G.G.); \\ sylwiagoras@gmail.com (S.G.) \\ 2 Department of Nanometrology, Faculty of Microsystem Electronics and Photonics, Wroclaw University of \\ Science and Technology, 50-372 Wroclaw, Poland; paulina.szymanowska@pwr.edu.pl (P.S.); \\ tomasz.piasecki@pwr.edu.pl (T.P.); teodor.gotszalk@pwr.edu.pl (T.G.) \\ * Correspondence: zuzanna.drulis-kawa@uwr.edu.pl
}

Received: 17 March 2020; Accepted: 4 April 2020; Published: 7 April 2020

\begin{abstract}
Bacterial biofilm prevention and eradication are common treatment problems, hence there is a need for advanced and precise experimental methods for its monitoring. Bacterial resistance to antibiotics has resulted in an interest in using a natural bacterial enemy-bacteriophages. In this study, we present the application of quartz tuning forks (QTF) as impedance sensors to determine in real-time the direct changes in Pseudomonas aeruginosa PAO1 biofilm growth dynamics during Pseudomonas phage LUZ 19 treatment at different multiplicities of infection (MOI). The impedance of the electric equivalent circuit (EEC) allowed us to measure the series resistance (Rs) corresponding to the growth-medium resistance (planktonic culture changes) and the conductance $(\mathrm{G})$ corresponding to the level of QTF sensor surface coverage by bacterial cells and the extracellular polymer structure (EPS) matrix. It was shown that phage impacts on sessile cells ( $G$ dynamics) was very similar in the 10-day biofilm development regardless of applied MOI $(0.1,1$ or 10). The application of phages at an early stage (at the sixth $\mathrm{h}$ ) and on three-day biofilm caused a significant slowdown in biofilm dynamics, whereas the two-day biofilm turned out to be insensitive to phage infection. We observed an inhibitory effect of phage infection on the planktonic culture (Rs dynamics) regardless of the MOI applied and the time point of infection. Moreover, the Rs parameter made it possible to detect PAO1 population regrowth at the latest time points of incubation. The number of phage-insensitive forms reached the level of untreated culture at around the sixth day of infection. We conclude that the proposed impedance spectroscopy technique can be used to measure the physiological changes in the biofilm matrix composition, as well as the condition of planktonic cultures in order to evaluate the activity of anti-biofilm compounds.
\end{abstract}

Keywords: Pseudomonas aeruginosa; phage LUZ19; biofilm; quartz tuning forks; impedance spectroscopy

\section{Introduction}

Bacterial biofilm is a complex and dynamically changing structure responding to the external environment. Microbial cell aggregates are surrounded by complex polymer substances (matrix) composed of proteins, polysaccharides, eDNA (extracellular DNA), glycolipids and fatty acids [1]. Bacteria inside the biofilm are not synchronized in terms of metabolic activity, and biofilm structure is highly heterogeneous in this respect [2,3]. The protection against unfavorable environmental conditions can be implemented by the extracellular polymer structure (EPS) production, increasing 
significantly the resistance to chemical compounds and the immune system response [4]. Biofilm is a long-term strategy for bacterial survival under stress conditions. Cells embedded in the consortia cause biomaterials dysfunction, chronic infections, are highly insensitive to treatment and pose a threat in industry or are responsible for biofouling [5]. The high insensitivity of biofilm to antibiotics forces the search for alternative treatment solutions. Bacteriophages turn out to be a convenient weapon against both planktonic and sessile forms of bacteria [3,6,7]. To evaluate the complex and sublime interactions of antibacterials and biofilm cells it was necessary to develop various measurement techniques.

Currently, there are several methods allowing the characterization of biofilm components. The colony count (CFU/mL) of sessile cells colonizing biofilm structure is commonly used. The crystal violet $(\mathrm{CV})$ assay is used for biomass measurement because the dye is non-specific and interacts with negatively charged moieties of cells and biofilm matrix [8]. Other staining techniques are more specific showing the dye interaction with different biofilm elements. The commonly used Live/Dead BacLight kit composed of $S Y T O^{\circledR}$ dyes with orange acridine or propidium iodide (Merck Millipore, Burlington, MA, USA), distinguishes live and dead cells [9]. Resazurin (Alamar Blue), XTT (2,3-bis-(2-methoxy-4-nitro-5-sulfophenyl)-2H-tetrazolium-5-carboxanilide) and TTC (2,3,5-triphenyl-2H-tetrazolium chloride) are another group of markers for determining metabolically active cells. Calcofluor white or FITC-labeled lectins are used to stain the glycoconjugate fraction of biofilm [10]. FITC also interacts with the amino residues of proteins and amino sugars [11,12]. For fast determination, it is possible to use Nile Red staining protocol [10]. These dyes can be detected spectrophotometrically, but also fluorometrically using microplate readers or CLSM microscopy [13].

Visualizations of biofilm development and its eradication by antibiotics, phages or other combined therapies are an inseparable group of methods used in biofilm research. Microscopy techniques based on transmission electron microscopy (TEM), scanning electron microscopy (SEM), atomic force (AFM) or confocal microscopy are often used [14,15]. However, this group of methods is quite expensive and requires the use of sophisticated microscopy tools and material preparation techniques.

There is also an interesting method for biofilm measurement based on interferometry spectroscopy allowing us to determine the changes in the diffusion rate through the biofilm matrix $[3,7,16,17]$.

Each method used for biofilm monitoring has both advantages and limitations. In most cases, they are based on mandatory material preparation techniques, and do not allow measurements of long-term biofilm growth dynamics under the culture conditions. Monitoring of changes in the biofilm structure (EPS and cells) in real-time is particularly difficult. In this paper, we present the application of the impedance spectroscopy (IS) based on the application of quartz tuning forks (QTFs) as an impedance sensor. We have already published the physical background of QTF system applications in biofilm mass measurements [15,18].

In this study, we present our impedance spectroscopy set up for the monitoring of electrical properties of biofilm structure and bacterial culture. Measuring conductance and, we were able to detect the changes in biofilm development as well as in planktonic cells propagation. The application of QTF impedance systems were tested and verified on a Pseudomonas aeruginosa PAO1 culture model treated with Pseudomonas lytic phage LUZ19.

\section{Materials and Methods}

\subsection{Bacterial Strain and Phages}

Pseudomonas aeruginosa PAO1 (ATCC 15692) was used as a model of biofilm-forming bacteria. Bacterial cells were stored at $-70{ }^{\circ} \mathrm{C}$ in Trypticase Soy Broth (TSB, Becton Dickinson and Company, Cockeysville, MD, USA) supplemented with 20\% glycerol (U.S. Merck Corporate Headquarters, Kenilworth, NJ, USA). For the experiments, strains were refreshed on Trypticase Soy Agar (TSA, Becton Dickinson and Company, Cockeysville, MD, USA) at $37^{\circ} \mathrm{C}$ for $18 \mathrm{~h}$. Pseudomonas LUZ19 podovirus equipped with polysaccharide depolymerase was kindly provided from the collection of the Laboratory of Gene Technology, KU, Leuven, Belgium. 


\subsection{Bacterial Growth Measured by the Colony Count}

P. aeruginosa PAO1 culture was incubated at $37^{\circ} \mathrm{C}$ in $1.5 \mathrm{~mL}$ of TSB medium with immersed quartz tuning forks (QTF). The biofilm and planktonic growth dynamics were measured by the classical colony forming units (CFU/mL) at specific time points: at 6, 12, 24, 48, 72, 96, 144, 192, 216 and $240 \mathrm{~h}$ of the experiment. QTFs covered with biofilm were transferred to eppendorf tubes containing $150 \mu \mathrm{L}$ of PS (physiological saline, Avantor Performance Materials, Gliwice, Poland). To liberate cells from the biofilms and to measure the cell counts, biofilm was disintegrated using the ultrasonic cleaning bath USC300TH with $45 \mathrm{kHz}$ frequency and $80 \mathrm{~W}$ power for $25 \mathrm{~min}$ for all sensors (VWR International Ltd., Lutterworth, Leicestershire, UK), released cells were plated on Trypticase Soy Agar plates (Becton Dickinson and Company, Cockeysville, MD, USA) and the colony count was evaluated after $18 \mathrm{~h}$ incubation at $37^{\circ} \mathrm{C}$. The colony count was done for phage treated and untreated samples.

\subsection{Phage Treatment}

Pseudomonas phage LUZ19 filtrate at $10^{9} \mathrm{pfu} / \mathrm{mL}$ (plaque forming units) was diluted to obtain a corresponding MOI 0.1, 1 and 10 used from biofilm treatment at different time points: 6, 48, $72 \mathrm{~h}$. Phages were suspended in TSB medium in $1.5 \mathrm{~mL}$ into which sensors with adhered biofilm were transferred. Phage LUZ19 at a concentration of $10^{9} \mathrm{pfu} / \mathrm{mL}$ and TSB alone were used as negative controls (no biofilm). All experiments were performed three times in at least 4 technical replicates.

\subsection{Bacterial Biofilm Growth Measured by the Impedance Method}

As was reported before [18], the Quartz Tuning Forks (QTFs) may be used as the impedance sensors for biofilm growth monitoring. The measured object may be the impedance sensor which electric impedance change with the changes of the environment in which it is placed.

To perform the impedance spectra, the IMP-STM32 impedance analyser was used [19] connected to a dedicated multiplexing measuring head with sockets for 24 QTF sensors (Figure 1). Such a setup allowed for the quasi-simultaneous impedance spectra measurements of 24 sequentially switched sensors in the frequency range from $100 \mathrm{mHz}$ to $100 \mathrm{kHz}$ in approximately 30-min intervals.

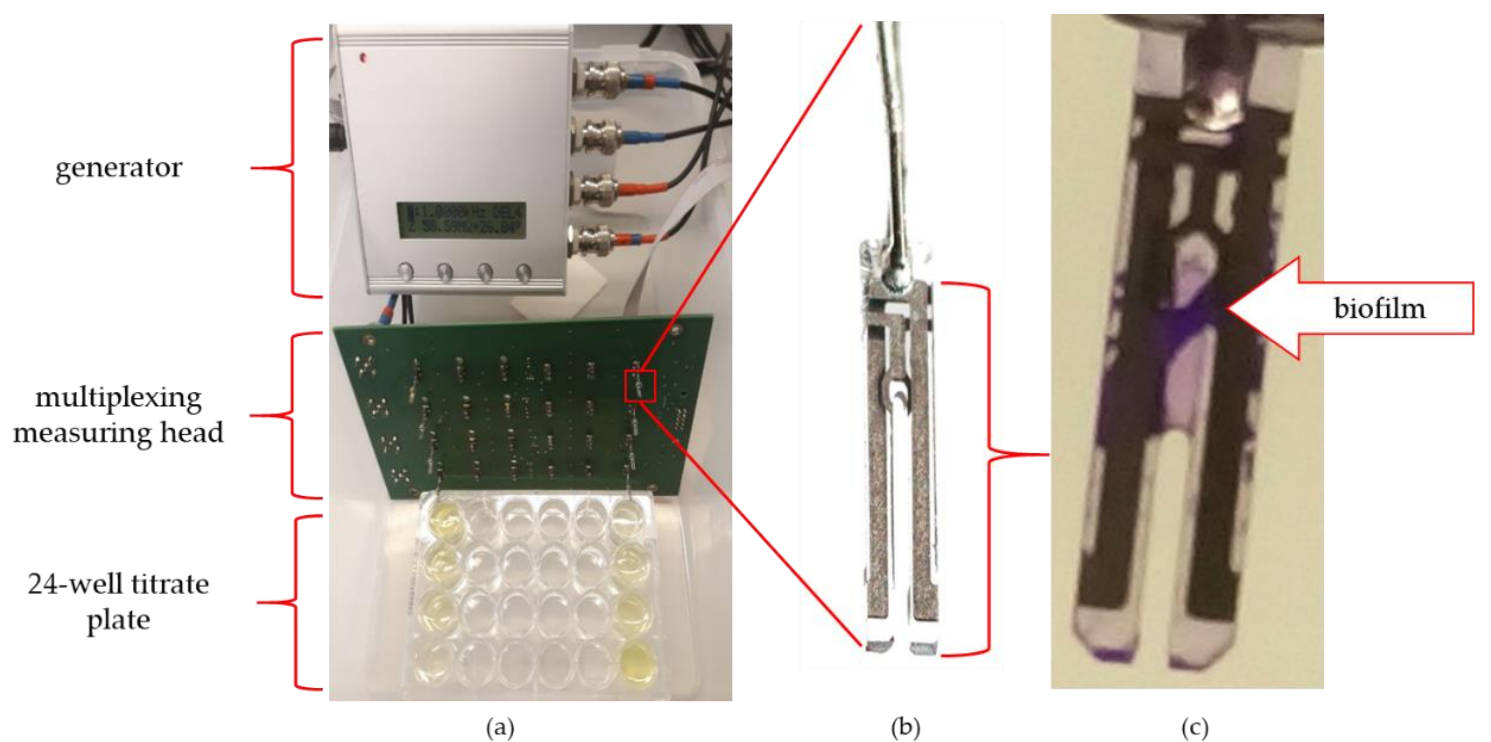

Figure 1. The measurement setup: (a) measurement system; (b) quartz tuning fork (QTF) senor; (c) biofilm covered QTF surface detected by crystal violet (CV) staining.

Prior to the experiments, the measuring head was sterilized with alcohol-based chemicals approximately $24 \mathrm{~h}$ before performing the experiments. Immediately before installing the sensors, 
the system was sterilized in a laminar chamber using ultraviolet for about $10 \mathrm{~min}$. The casings were removed from the QTF before the experiment and the sensors were rinsed with isopropanol (Avantor Performance Materials, Gliwice, Poland), which was evaporated at room temperature. A plate with bacterial cultures was prepared for the ready set of 24 sensors. Refreshed bacterial cultures were diluted in TSB to an $\mathrm{OD}_{550}$ (optical density) equal to 0.2 . The culture of $10^{6} \mathrm{CFU} / \mathrm{mL}$ was suspended in $1.5 \mathrm{~mL}$ in 24 wells culture plate (VWR International, LLC Radnor Corporate Centre, Radnor, PA, USA) and incubated at $37^{\circ} \mathrm{C}$ for $240 \mathrm{~h}$. Pseudomonas phages LUZ19 filtrate at MOI $0.1,1$ or 10 was added at 6,48 and $72 \mathrm{~h}$ of incubation. Each experiment was performed in triplicate with four technical repetitions $(N=12)$.

\subsection{Impedance Spectra Analysis}

The electrical impedance $Z=R+j X$ expressed in ohms $(\Omega)$ is the complex measure of the object's electrical response to the alternating voltage excitation, where $R$ is a resistance, $X$ is a reactance and $j$ is the imaginary unit. The technique in which impedance spectra, that is the representation of such responses measured over a wide range of frequencies, is called the impedance spectroscopy [20]. Results of such a measurement may be also presented as the complex admittance $Y$ expressed in siemens $(S)$, where real and imaginary parts are conductance $G$ and susceptance B.

A typical impedance spectra analysis method is fitting them with the impedance of the electric equivalent circuit (EEC) using dedicated software (for example, Scribner ZView that was used in presented research). Two EECs were used (Figure 2a,b).

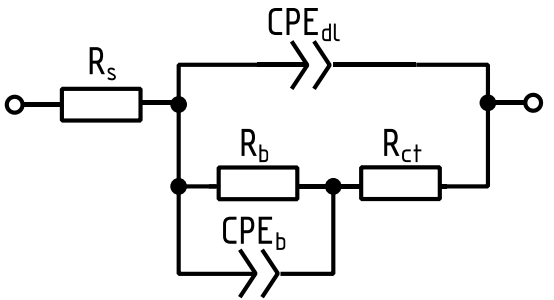

(a)

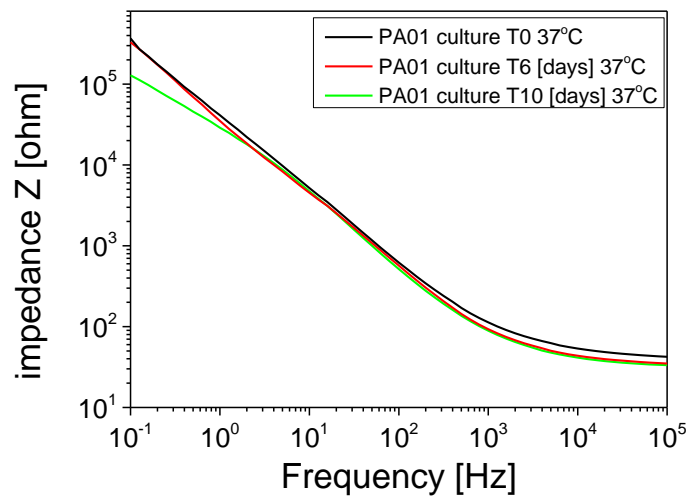

(c)

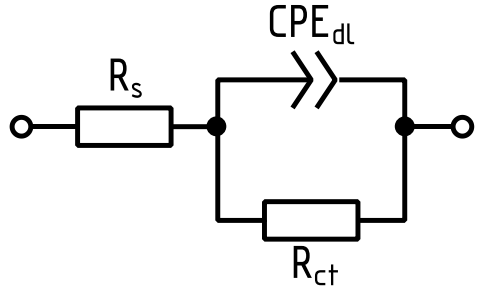

(b)

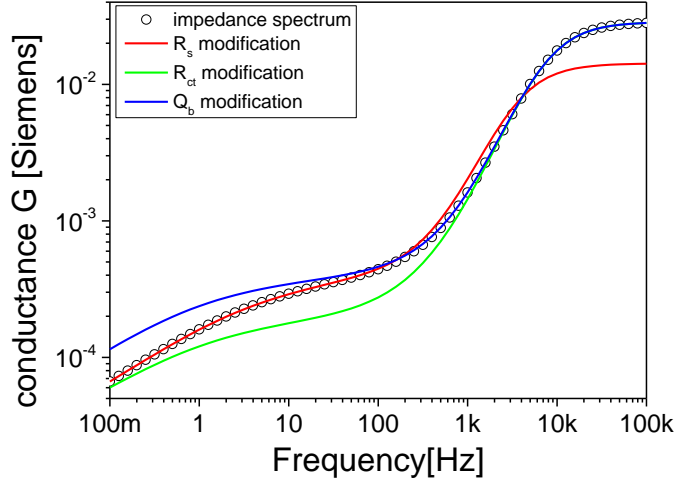

(d)

Figure 2. Primary (a) and simplified (b) electric equivalent circuits (EECs) used for the analysis of measured impedance spectra; (c) impedance spectra of quartz tuning forks (QTF) in P. aeruginosa PAO1 10-days biofilm culture, and the influence of EEC parameters change to the shape of conductance spectrum (d). 
These consisted of resistors and constant phase elements (CPE). CPE is the element frequently used in EEC modelling. Its impedance is equal $Z_{C P E}=\frac{1}{Q(2 \pi j f)^{T}}$, where $Q$ and $T$ are CPE parameters and $\mathrm{f}$ is frequency. Its components relate to: Rs, series resistance which corresponds mainly to the growth medium resistance; CPEdl, electric double layer capacitance between the growth medium and electrodes; Rct, charge transfer resistance; $\mathrm{Rb}$ and $\mathrm{CPEb}$, resistance and capacitance of the objects adhered to the electrode surface (mostly cells and biofilm).

Most of the fits were done with primary EEC however, in some experiments, the influence of $\mathrm{Rb}$ and $\mathrm{CPEb}$ on the measurement spectrum was not measurable. In these cases the simplified EEC gave better-fitting results. The optimal model was chosen based on the fit quality $\left(\chi^{2}\right)$ and the fitting error of EEC components. In simplified analyses after EEC modelling, the use of Rs and Qb parameters was proposed. Biologically, the changes in Rs parameter should be understood as an increase in the number of planktonic forms in the medium surrounding the QTF sensor [18]. The second important parameter of the EEC model is $\mathrm{Q}_{\mathrm{b}}$ (electrical conductivity) measured at $100 \mathrm{mHz}$. The biological equivalent of changes for the conductance $\left(Q_{b}\right)$ is the adhesion of bacterial cells to the sensor surface as well as the formation or degradation of the biofilm matrix. The analysis of the frequency ranges at which the most important EEC components influence the impedance spectrum shape allowed a simplification of the biofilm state assessment. The plot shown in Figure $2 \mathrm{~d}$ was created by simulating the conductance spectrum of the EEC fitted to one of the preliminary results (dots) and with $R_{s}, R_{c t}$ and $Q_{b}$ changed twofold. As may be observed, a change in $R_{s}$ altered the high-frequency part of the spectrum while $Q_{b}$ influences the low-frequency part. Therefore the Rs variations may be estimated by the real part of the impedance measured at $100 \mathrm{kHz}$. As the initial value of such a parameter varied between sensors it was normalized by its value at the $3 r d h$ of the experiment yielding $R_{100 k \_n o r m ~}$ presented in the Results section. Similarly, the $\mathrm{Q}_{\mathrm{b}}$ variations were estimated by the variations of the conductance (that is the real part of the inverse of the impedance) $\mathrm{G}_{100 \mathrm{~m}}$ measured at $100 \mathrm{mHz}$. The similarity between parameters obtained using the EEC and simplified analysis is shown in Figure 6a,b.

\subsection{Biofilm Monitoring by Scanning Electron Microscopy (SEM)}

Sensors covered with bacterial biofilm were transferred to eppendorf tubes with PS to remove unadhered cells. Next, the sensors were placed in a series of increasing ethanol concentrations: $60 \%$, $70 \%, 80 \%, 96 \%$ and the remaining water content was evaporated in vacuum conditions. After $24 \mathrm{~h}$ the preparations were transferred to a vacuum evaporator and the silver conductive layer spraying process was carried out. Biofilm presence on QTF surface was visualized by SEM at $30 \times, 100 \times$ and 3000× magnifications (Tesla BS 300, Brno, Czechoslovakia).

\subsection{Statistical Analyses}

The statistical analysis was performed for three independent experiments and four technical repetitions $(N=12)$ using a one-way ANOVA. To compare the differences between variances, Levene's statistical method was applied. Results were considered significant at $p<0.05$ value.

\section{Results}

\subsection{Biofilm and Planktonic Population Growth Measured by Standard Colony Count and SEM}

The dynamics of PAO1 biofilm growth on quartz surface was determined by a standard colony count for both phage treated and untreated culture (Figure 3). The phage LUZ19 at MOI = 1 was applied at three different culture times (marked with colored arrows in Figure 3), corresponding to the 6th, 48th and 72nd h of incubation. Cell counts were measured starting from $6 \mathrm{~h}$ until 10 days of incubation. In the untreated biofilm experiment, an increase in the number of cells is observed, with a maximum of about $10^{8} \mathrm{CFU} / \mathrm{mL}$ for $48 \mathrm{~h}$ incubation (black curve in Figure 3a). Further incubation gradually reduces the population up to around $10^{6} \mathrm{CFU} / \mathrm{mL}$ on the tenth day of the experiment. Phage application at the 6 th $\mathrm{h}$ inhibited the propagation of sessile cells for one day, and further, the biofilm 
population started growing up to the maximum of $10^{9} \mathrm{CFU} / \mathrm{mL}$ after four days of incubation (red curve in Figure 3a). The application of phage LUZ19 at MOI $=1$ at 48 and $72 \mathrm{~h}$ (blue and green curves in Figure 3a) caused a decrease in the number of sessile cells at the level of 2-3 logs followed by the population regrowth and a final count of around $10^{6} \mathrm{CFU} / \mathrm{mL}$. The permanent exposition of biofilm to phage infection resulted in the fluctuation of the cell number embedded in biofilm matrix from $10^{9}-10^{8} \mathrm{CFU} / \mathrm{mL}$ to $10^{5} \mathrm{CFU} / \mathrm{mL}$. Prolonged biofilm cultures infected with phage LUZ19 stabilized the colony count at around $10^{6} \mathrm{CFU} / \mathrm{mL}$ after 10 days of incubation regardless of the time point of phage infection.

LUZ19 phage infection at the beginning of planktonic culture incubation $(6 \mathrm{~h})$ caused the drop of 3 logs when measured at a 24th of the incubation time. The next day, a clear regrowth of planktonic forms to the level of $10^{7} \mathrm{CFU} / \mathrm{mL}$ was observed and gradually increased up to a maximum of $10^{9} \mathrm{CFU} / \mathrm{mL}$ at the 96th $\mathrm{h}$ of the experiment. A similar dynamic was noticed for planktonic forms, where the phage was applied at the second and third day of incubation, leading to a sudden drop of 3-4 logs in CFU after one day of phage propagation forwarded by the fast phage-insensitive population regrowth with the maximum of $10^{8} \mathrm{CFU} / \mathrm{mL}$ (blue and green curve in Figure $3 \mathrm{~b}$ ). All phage-treated planktonic cultures stabilized the colony count at around $10^{8} \mathrm{CFU} / \mathrm{mL}$ after 10 days of incubation.

(a)
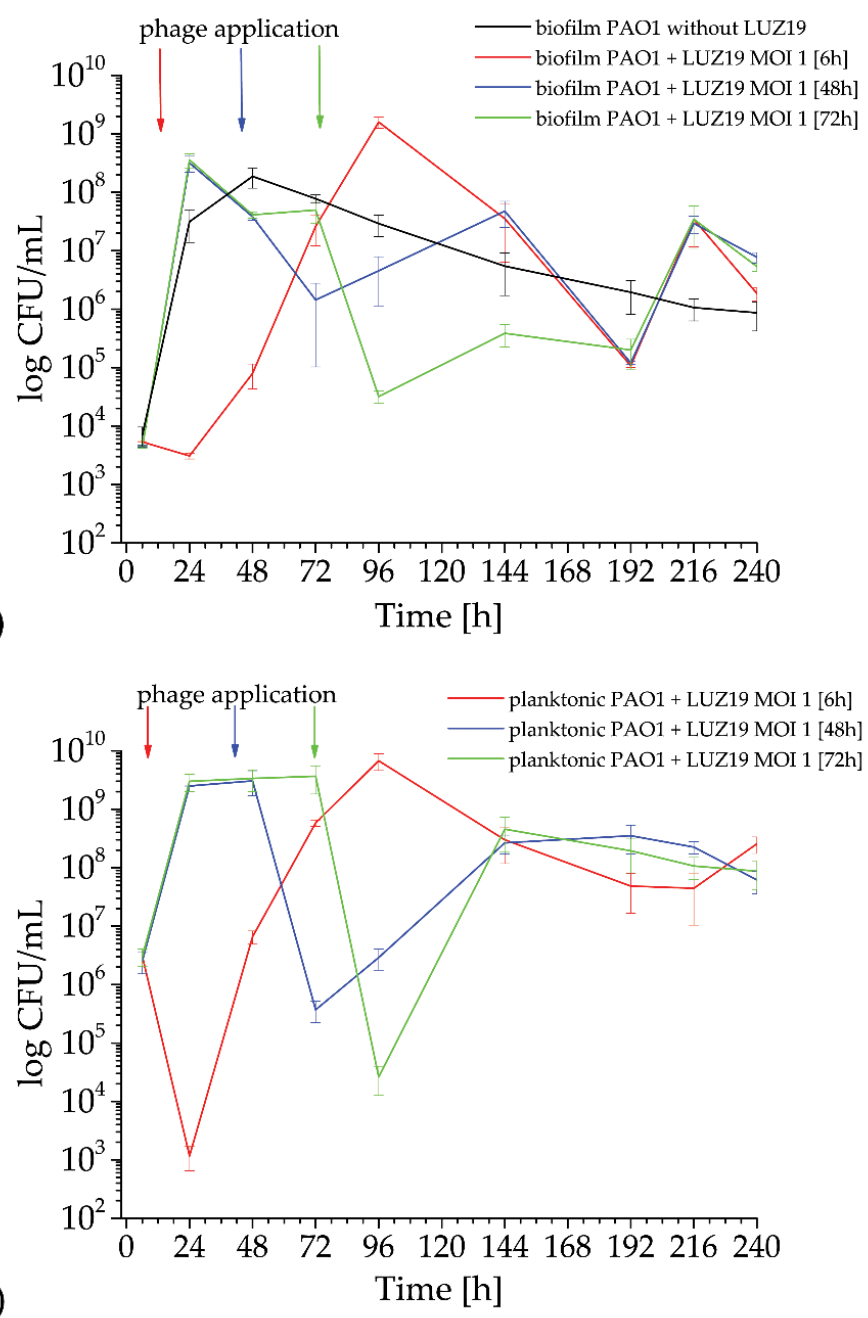

Figure 3. The colony count of P. aeruginosa PAO1 biofilm growth on QTF surface (a) and planktonic population (b) after phage LUZ19 infection. The error bars indicate the standard deviation. The results are presented as the means $\mathrm{CFU} / \mathrm{mL} \pm \mathrm{SD}$ of three independent experiments in triplicate. 
To confirm the presence of biofilm formation on the surface of the QTF sensor, we performed the analysis using a scanning electron microscope (SEM). As part of the control experiment, the sensors were suspended in TSB broth without bacteria (Figure 4). Microscopic images show the structure of the sensor surface. It consists of evenly distributed quartz crystals making a pyramidal form. Bright elements appearing are not bacterial cells but likely derive from diluents or result from post-production sensor impurities.

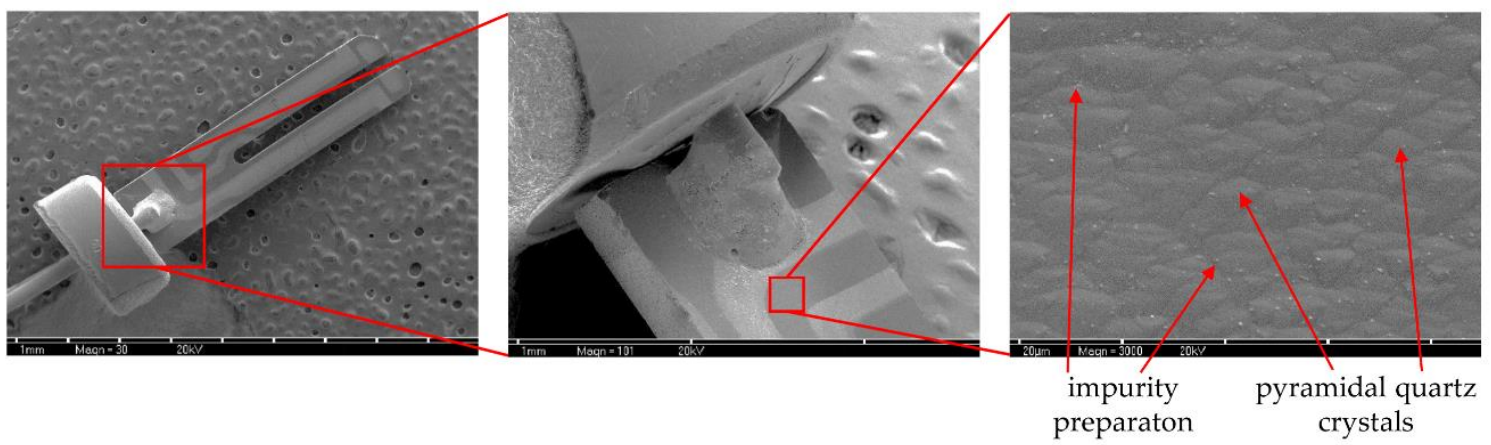

Figure 4. Visualization of QTF sensor surface immersed in TSB medium (without bacterial cells) at magnifications of $30 \times$ (left part) $100 \times$ (middle) and 3000× (right part), respectively.

Time points of biofilm visualization with SEM (Figure 5) were selected based on changes in the $\mathrm{G}_{100 \mathrm{~m}}$ parameter (see paragraph 3.2). Figure 5 is divided into upper and lower panels, and sensors are presented with untreated biofilm after phage LUZ19 application at the 6th, 48th and 72th $\mathrm{h}$ of incubation. The upper panel shows the tuning fork ring at $30 \times$ magnification, while the lower panel shows the QTF quartz surface at $3000 \times$ magnification. Under the panel, the $\mathrm{CFU} / \mathrm{mL}$ values corresponding to selected time points are added. In the case of QTF incubated in phage untreated PAO1 culture, the pyramidal quartz crystals are completely obscured by biofilm components and the entire surface is covered by a layer of EPS matrix, and EPS matrix fragments are clearly visible on days 8 (196 h) and $10(240 \mathrm{~h})$ of biofilm incubation. The matrix and densely arranged bacterial cells in anchored large groups are visible at a higher magnification. At the end of the experiment $(240 \mathrm{~h})$, the microscopic visualization shows a reduction in EPS thickness and cells are no longer fully covered with a thick matrix layer. In contrast, there were no significant changes in SEM pictures between phage-treated biofilm and only a thin EPS layer was detected for all samples. The differences in the amount of EPS/matrix covering the sensor surface were seen for $196 \mathrm{~h}$ and $240 \mathrm{~h}$ biofilm compared to untreated samples. Considering the results of the colony count and SEM analyses, it might be assumed that the differences in biofilm imaging seen in microscopy analysis are rather related to the amount of the EPS and not to the number of sessile cells embedded in biofilm structure. 
$\mathrm{T}=144 \mathrm{~h}$

control - without phage LUZ19

phage LUZ19 at 6h

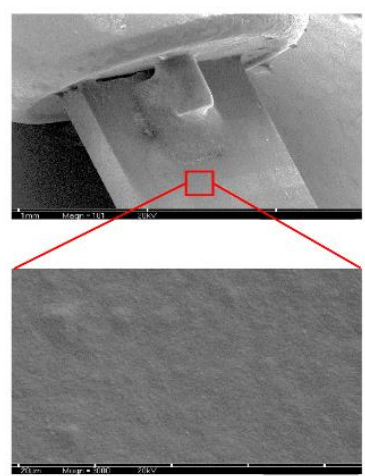

$\mathrm{CFU} / \mathrm{mL}=3.5^{*} 10^{7}$ phage LUZ19 at $48 \mathrm{~h}$

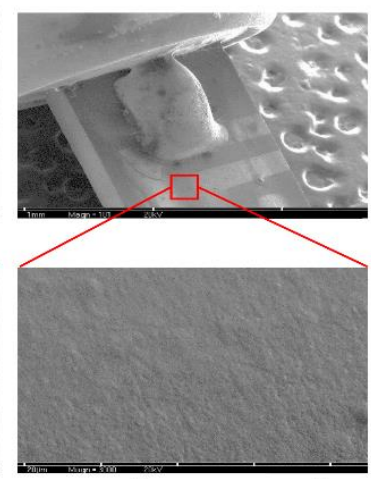

$\mathrm{CFU} / \mathrm{mL}=4.7^{*} 10^{7}$ phage LUZ19 at $72 \mathrm{~h}$

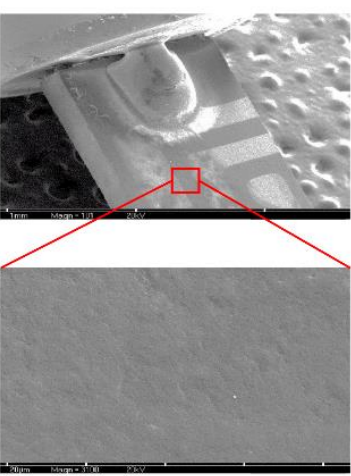

$\mathrm{CFU} / \mathrm{mL}=3.8^{*} 10^{5}$

\section{$\mathbf{T}=192 \mathrm{~h}$}

control - without phage LUZ19

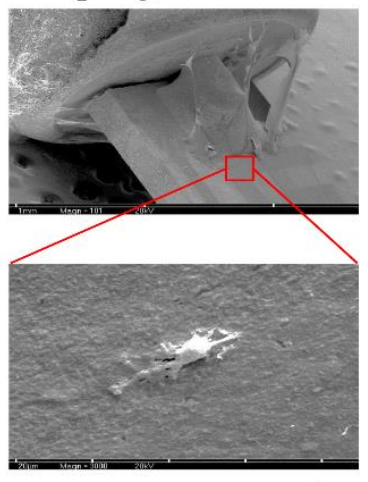

$\mathrm{CFU} / \mathrm{mL}=2.0^{*} 10^{6}$ phage LUZ19 at $6 \mathrm{~h}$

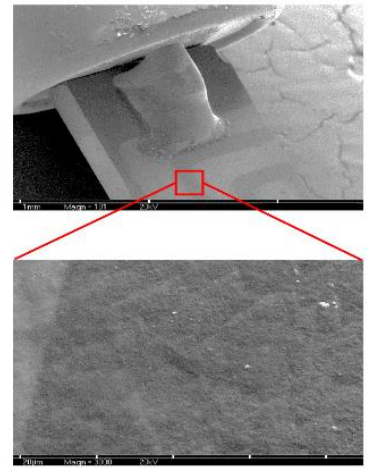

$\mathrm{CFU} / \mathrm{mL}=1.0^{*} 10^{5}$ phage LUZ19 at $48 \mathrm{~h}$

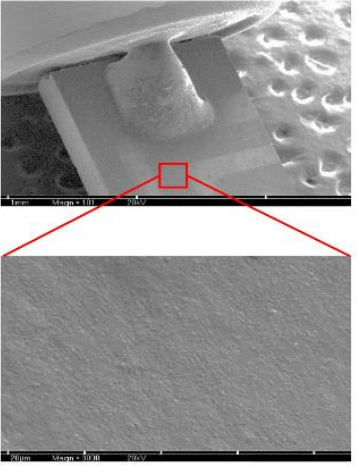

$\mathrm{CFU} / \mathrm{mL}=1.2^{*} 10^{5}$ phage LUZ19 at 72h

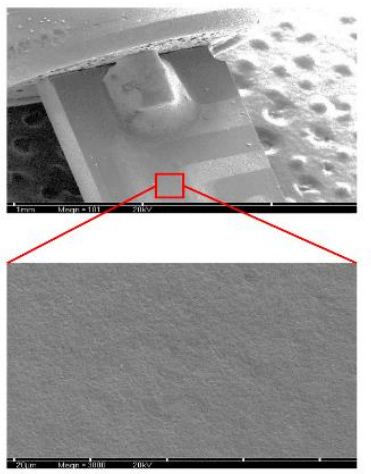

$\mathrm{CFU} / \mathrm{mL}=2.0^{*} 10^{5}$

\section{$\mathrm{T}=240 \mathrm{~h}$}

control - without phage LUZ19
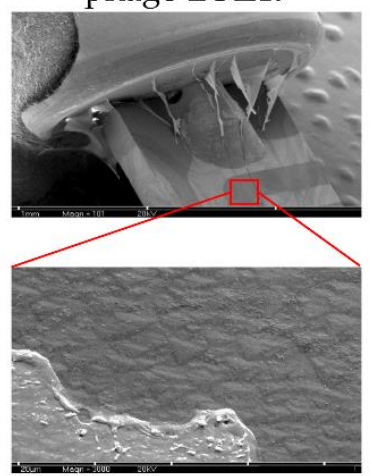

$\mathrm{CFU} / \mathrm{mL}=8.7^{*} 10^{5}$

phage LUZ19 at $6 \mathrm{~h}$

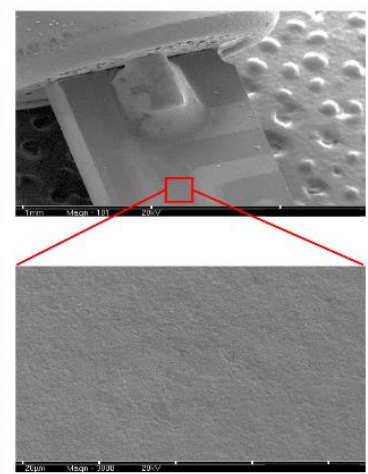

$\mathrm{CFU} / \mathrm{mL}=1.8^{*} 10^{6}$ phage LUZ19 at $48 \mathrm{~h}$
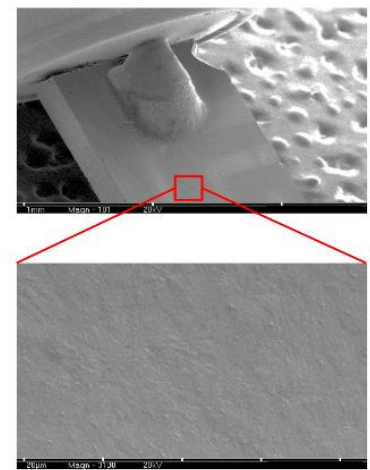

$\mathrm{CFU} / \mathrm{mL}=7.7^{*} 10^{6}$ phage LUZ19 at $72 \mathrm{~h}$

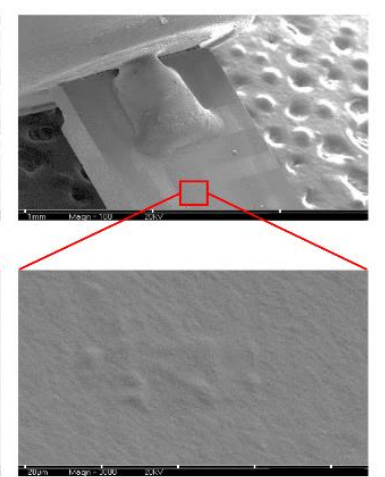

$\mathrm{CFU} / \mathrm{mL}=5.3^{*} 10^{6}$

Figure 5. Visualization of QTF sensors at the 144th, 192nd, and 240th $\mathrm{h}$ of untreated and phage LUZ19 treated biofilm at MOI = 1 (from left to right, added at the 6th, 48th and 72nd h of culture, respectively). The upper panel shows the tuning fork ring at 30× magnification. The bottom panel enlarged 3000× QTF surface. The bottom panel presents the $\mathrm{CFU} / \mathrm{mL}$ values corresponding to selected time point. 
3.2. Standarization of Real-Time Monitoring of P. aeruginosa PAO1 Biofilm Growth under Control Conditions Using QTF Impedance Spectroscopy

The standardization of a real-time monitoring of P. aeruginosa PAO1 biofilm growth using QTF impedance spectroscopy was performed (Figure 6). The initial drop of resistance $\mathrm{R}_{100 \mathrm{k}}$ was caused by the temperature stabilization of growth medium.

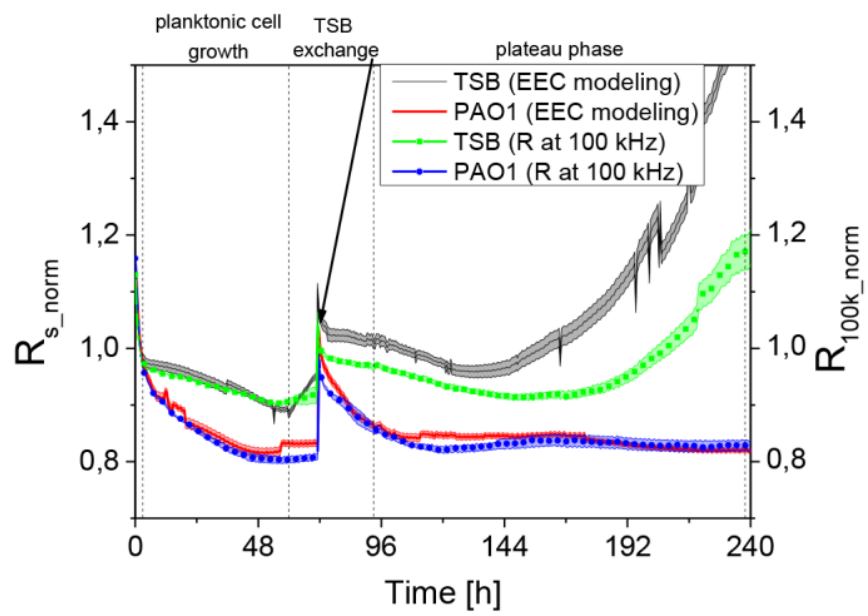

(a)

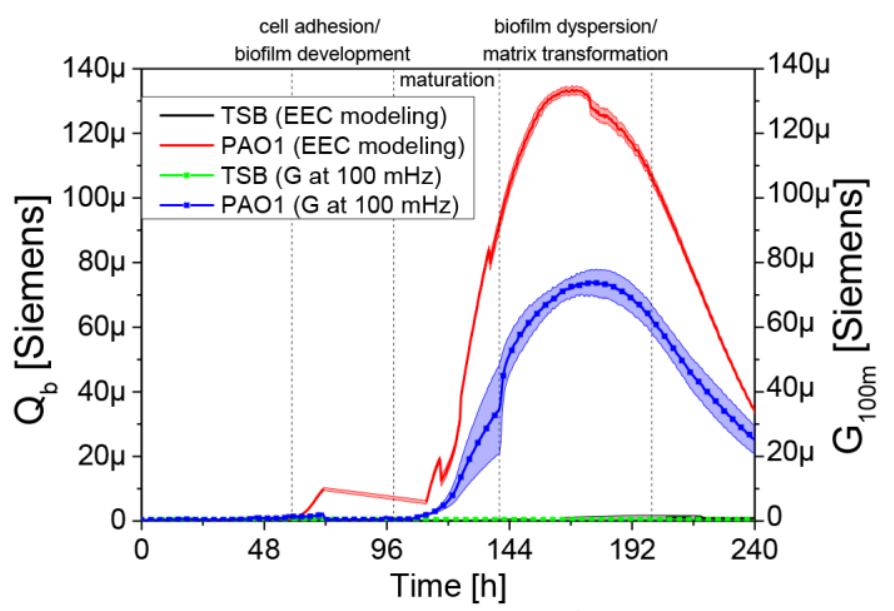

(b)

Figure 6. (a) Comparison of the normalized resistance parameter measured by the method using EEC

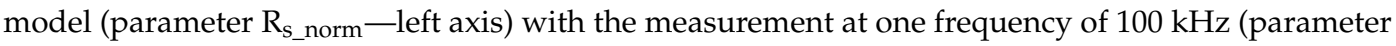
$\mathrm{R}_{100 \mathrm{k} \text { norm }}$-right axis); (b) Comparison of the conductance parameter for the method using EEC model (parameter $\mathrm{Qb}$-left axis) the measurement at $100 \mathrm{mHz}$ (parameter $\mathrm{G}_{100 \mathrm{~m}}$-right axis).

Starting from the sixth $h$ of the experiment, the $R_{100 k}$ value was gradually decreasing for up to $48 \mathrm{~h}$ for the sensor immersed in bacterial culture compared to the TSB control. This change is connected to bacteria growth near the sensor surface. After transferring to a new TSB medium at $72 \mathrm{~h}$ of incubation, there was a peak in the resistance value as the result of the stabilization of the sensor in a new medium followed again by the planktonic forms propagation until a stable level of $R_{100 k \_n o r m}$ was seen at the same level as before the exchange of medium $(\sim 110-120 \mathrm{~h})$. On the fourth

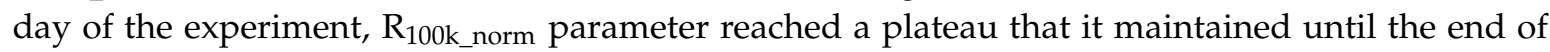
experiment. For QTF sensors immersed in TSB medium, a temperature stabilization of about three $h$ 
was observed. Next, the sensor was functionalized and metallic electrodes underwent an oxidation process which is expressed by a slight decrease in the $\mathrm{R}_{100 \mathrm{k} \_n o r m}$ parameter. The sensor transfer into fresh medium at $72 \mathrm{~h}$ caused a similar peak formation as that for the tested sample. At the later stages in the TSB control experiment (after $144 \mathrm{~h}$ ), an increase in resistance was observed over time, which can be equated with the physicochemical changes of the medium and the further oxidation processes on the QTF surface. The second measured parameter $\left(Q_{b}\right)$ was used to assess the electrical conductivity of biological objects adhering to the sensor surface. Fresh medium introduction at $72 \mathrm{~h}$ lead again to $\mathrm{Q}_{\mathrm{b}}$ plateau until $\sim 110-120 \mathrm{~h}$. There was a relationship between the resistance parameter $\left(\mathrm{R}_{\mathrm{s} \_ \text {norm }}\right)$ and $\mathrm{Q}_{b}$ representing the conductance parameter. After reaching the plateau level in Rs, the electrical conductivity $\mathrm{Q}_{b}$ was increasing. The $\mathrm{Q}_{\mathrm{b}}$ grew from $\sim 110-120 \mathrm{~h}$ to $\sim 180 \mathrm{~h}$ of experiment, reaching the value of $130 \mu$ Siemens. That increase can be explained by bacterial cell adhesion to the sensor surface and by formation of the conductive EPS matrix. After $180 \mathrm{~h}$ of incubation, the $\mathrm{Q}_{\mathrm{b}}$ curve was going down and those changes were identified as the dispersion of biofilm or modifications and degradation of matrix presented as the depletion of electrical charge in the broth culture. Later in the text, the $Q_{b}$ parameter is replaced by a $\mathrm{G}_{100 \mathrm{~m}}$ conductance parameter derived for simplified EEC as explained in the Materials and Methods Section 2.5. The dynamics of conductance changes in $\mathrm{G}_{100 \mathrm{~m}}$ identified with the creation and reconstruction of EPS matrix, requiring the need for visualization using a scanning microscope, which directly proved the correlation of the electrical parameter with the IS method and visualization in SEM. Conductance changes in $\mathrm{G}_{100 \mathrm{~m}}$ have been shown to be strongly associated with the reduction of EPS matrix in longer biofilm cultures.

\subsection{Monitoring of PAO1 Biofilm Growth during Pseudomonas Phage LUZ19 Treatment Measured by Impedance Spectroscopy}

All control experiments, by standardizing experimental conditions, enabled the application of impedance measurements using the QTF sensor platform to monitor the dynamics of biofilm growth during the treatment with phage LUZ19. Additional negative controls consisted of $10^{9} \mathrm{pfu} / \mathrm{mL}$ of phage LUZ19 in TSB medium (Figure 7).

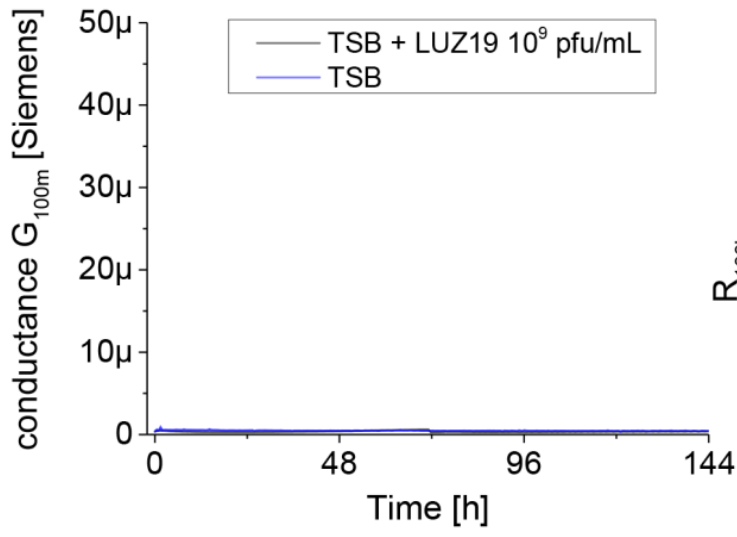

(a)

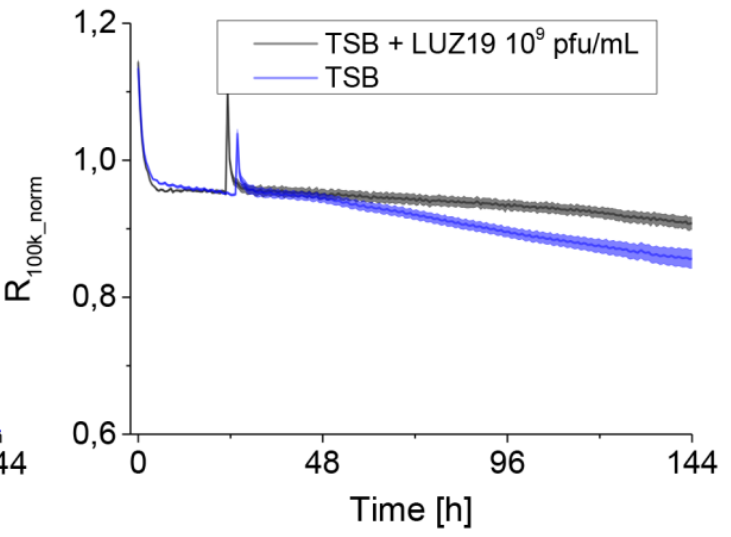

(b)

Figure 7. The changes in conductance $\mathrm{G}_{100 \mathrm{~m}}$ (left panel) and resistance $\mathrm{R}_{100 \mathrm{k} \_n o r m}$ (right panel) measured by QTF sensors for negative control consisted of phage LUZ19 and TSB medium. Data are expressed as mean conductance $\mathrm{G}_{100 \mathrm{~m}} \pm \mathrm{SD}$ and $\mathrm{R}_{100 \mathrm{k} \_ \text {norm }}$ of four independent experiments.

The measured parameters of normalized resistance at $100 \mathrm{kHz}$ for TSB curves and phage LUZ19 ran similarly over the course of the seven-day experiment. For the $\mathrm{G}_{100 \mathrm{~m}}$ parameter, no conductivity disturbances of QTF sensors were also observed, which confirmed the possibility of biofilm dynamics measurement [18]. For biofilm treatment, phage LUZ19 was used at three MOIs (0.1, 1 and 10) and different times of administration. The MOI value was calculated based on the CFU value presented in Figure 3. The phage application points corresponded to the 6th, 48th and 72nd $h$ of culture 
incubation to obtain the effect of adhesion prevention and biofilm eradication at different time points. The experiments were carried out for 10-days in the culture. Two electrical parameters of the culture were measured in real-time to determine biofilm formation dynamics. The first parameter was the conductance $\mathrm{G}_{100 \mathrm{~m}}$ associated with bacterial cell attachment to the surface of the QTF sensor and the growth of sessile forms. In parallel, the series resistance (Rs) of the medium showed the changes in the medium composition and planktonic cells growth. The dynamics of electrical parameters of PAO1 biofilm culture treated with phage LUZ19 at different MOIs were compared to a non-infected control.

The results are presented as conductance (left panel Figures 8-10) and normalized series resistance (right panel Figures 8-10). A set of 24 sensors covered by bacterial biofilm was transferred at selected time points to a new plate containing TSB broth with phage LUZ19 suspended at appropriate MOIs. The control non-treated PAO1 culture was also transferred to new TSB medium simultaneously as the treated sample. The dynamics of the $\mathrm{G}_{100 \mathrm{~m}}$ conductance parameter changed after phage application (Figures 8-10, left panels) and were very similar regardless of MOI applied. We noticed that the moment of phage administration largely determined the course of curves for measuring electrical parameters.

When phage was administered at six $\mathrm{h}$ of culture incubation, a strong inhibition in biofilm growth was observed regardless of MOI applied (Figures $8 \mathrm{a}, 9 \mathrm{a}$ and $10 \mathrm{a}$ ). The $\mathrm{G}_{100 \mathrm{~m}}$ parameter for PAO1 control began to increase at around $100 \mathrm{~h}$ into the experiment, reaching $20 \mu$ Siemens after $220 \mathrm{~h}$, while the conductance of phage-infected culture was at 3-5 $\mu$ Siemens up to 10 days into the experiment. The propagation of planktonic cells was slowed down when phage was applied at six $\mathrm{h}$ of culture incubation (Figures $8 \mathrm{~b}, 9 \mathrm{~b}$ and $10 \mathrm{~b})$. The resistance parameter $\left(\mathrm{R}_{100 \mathrm{k} \_n o r m}\right)$ made up the control culture at around the 60th and the 100th $\mathrm{h}$ of incubation for MOI 0.1 and MOI 1-10, respectively. Other results were obtained after LUZ19 application at $48 \mathrm{~h}$ of the culture. Biofilm was not sensitive to phage therapy regardless of applied phage concentration in the culture medium. The maximum conductance was observed around 220, 168 and 192 for MOI 0.1, 1 and 10, respectively, corresponding to the conductivity of around $20 \mu$ Siemens. The analysis of planktonic forms via serial resistance $R_{100 k}$ norm showed similar dynamics in generating phage-resistant forms regardless of MOI. The biofilm infection at $48 \mathrm{~h}$ after the start of the experiment generated similar cell regrowth regardless of applied LUZ19 concentrations, reaching a plateau phase by about the 144th $\mathrm{h}$.

There was a delay in biofilm formation compared to the control PAO1 when phage was introduced at the $72 \mathrm{nd} h$. The conductance dynamics was MOI independent, reaching the maximum of $30 \mu$ Siemens. The biofilm cultures on the sensors were carried out in stationary conditions before being transferred to the medium containing phage LUZ19. It is likely that the biofilm EPS at 72-h biofilm differs in the composition compared to the 48-h one, making the sessile cells sensitive to phage exposition. In Figures $8 \mathrm{e}, 9 \mathrm{e}$ and $10 \mathrm{e}$ a shift in the conductivity $\mathrm{G}_{100 \mathrm{~m}}$ increase was observed and the maximum was reached at about $230 \mathrm{~h}$, for each MOI. In this case, very similar fluctuations were found within the parameter characterizing planktonic forms. The $\mathrm{R}_{100 \mathrm{k} \_n o r m}$ curves reached a plateau phase of about $192 \mathrm{~h}$ for each MOI tested (Figures 8f, $9 \mathrm{f}$ and 10f). 


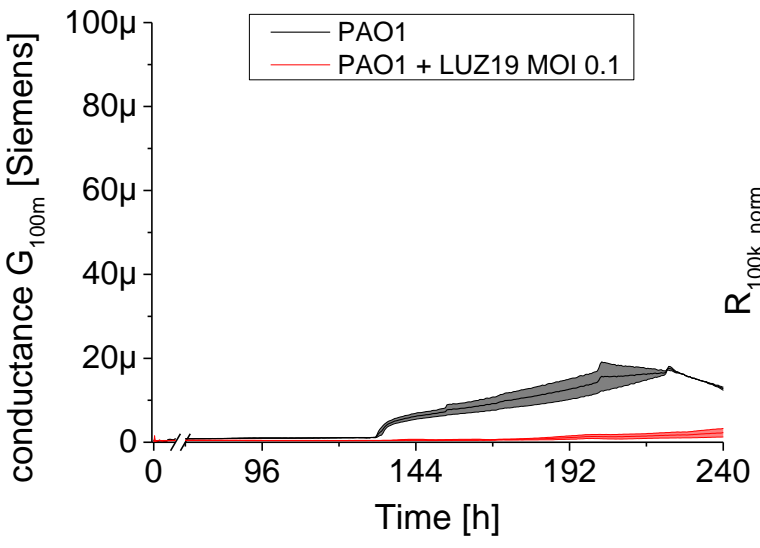

(a)

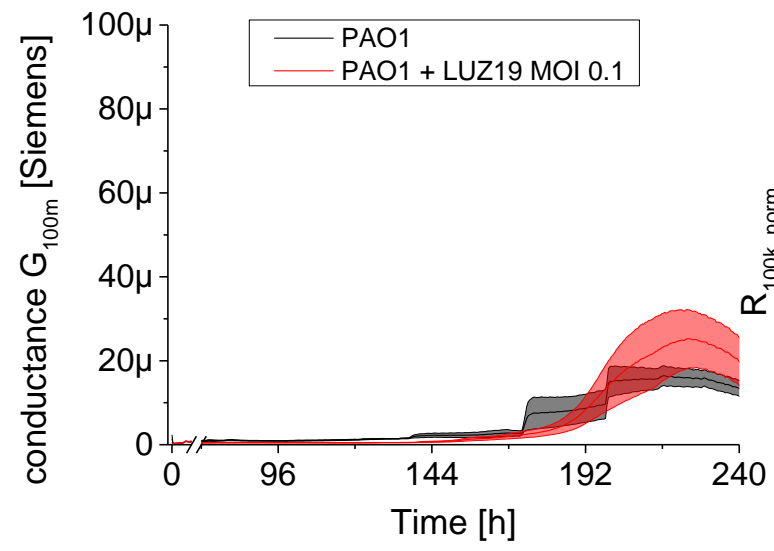

(c)

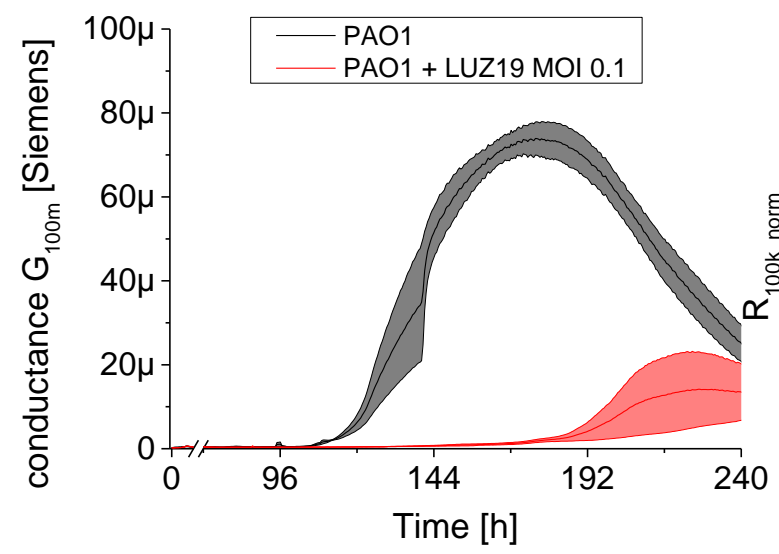

(e)

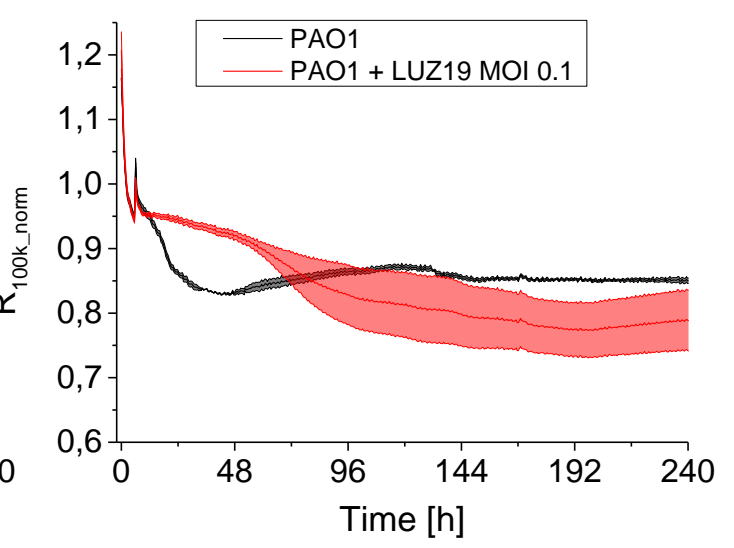

(b)

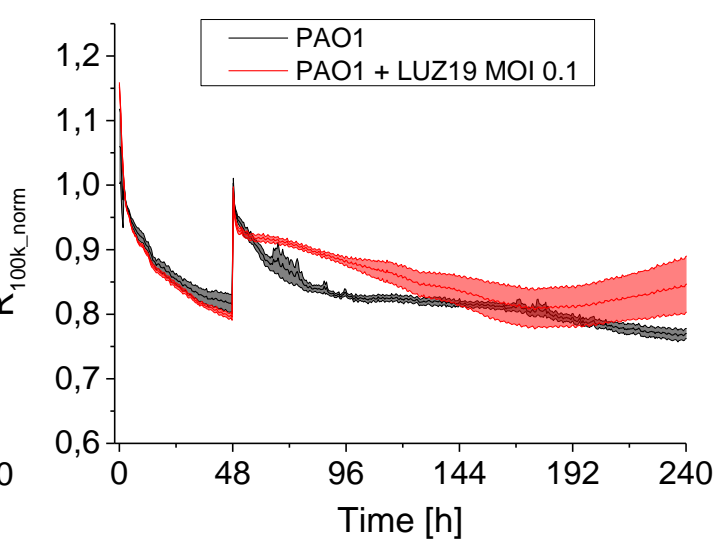

(d)

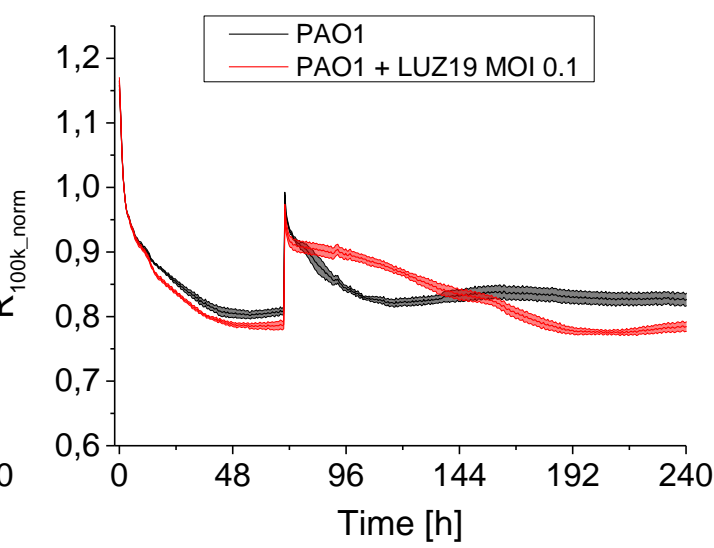

(f)

Figure 8. The changes in conductance $\mathrm{G}_{100 \mathrm{~m}}$ (left panel) and resistance $\mathrm{R}_{100 \mathrm{k} \_n o r m}$ (right panel) measured by QTF sensors during phage LUZ19 treatment of PAO1 biofilm at MOI $=0.1$. Phage was applied at the 6th $(\mathbf{a}, \mathbf{b}), 48$ th $(\mathbf{c}, \mathbf{d})$ and 72 nd $(\mathbf{e}, \mathbf{f})$ hour of biofilm culture. In the right panel, for each variant, a peak appears, which is associated with the transfer of QTF sensors to new medium containing an appropriate concentration of viral particles. Data are expressed as mean of conductance $\mathrm{G}_{100 \mathrm{~m}} \pm \mathrm{SD}$ and resistance $R_{100 k \_n o r m}$ from three independent experiments. 


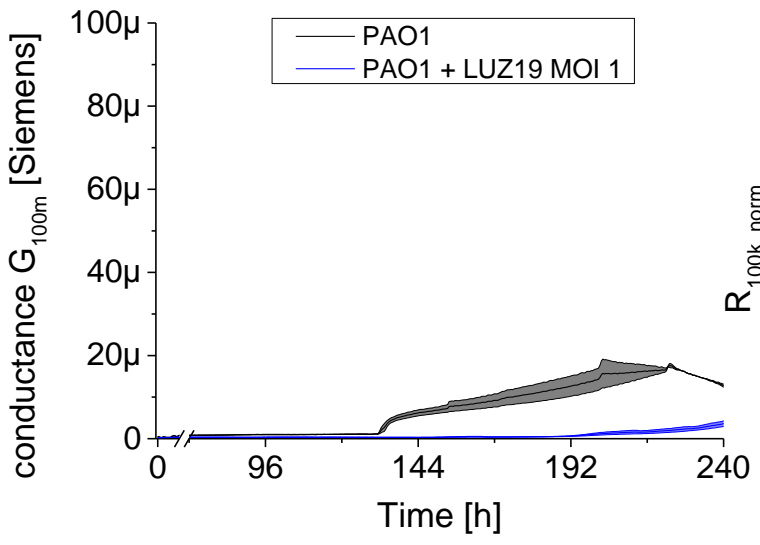

(a)

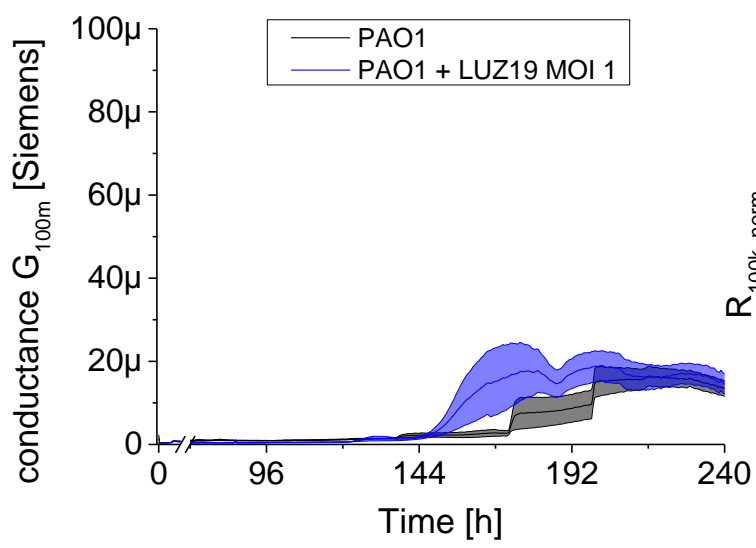

(c)

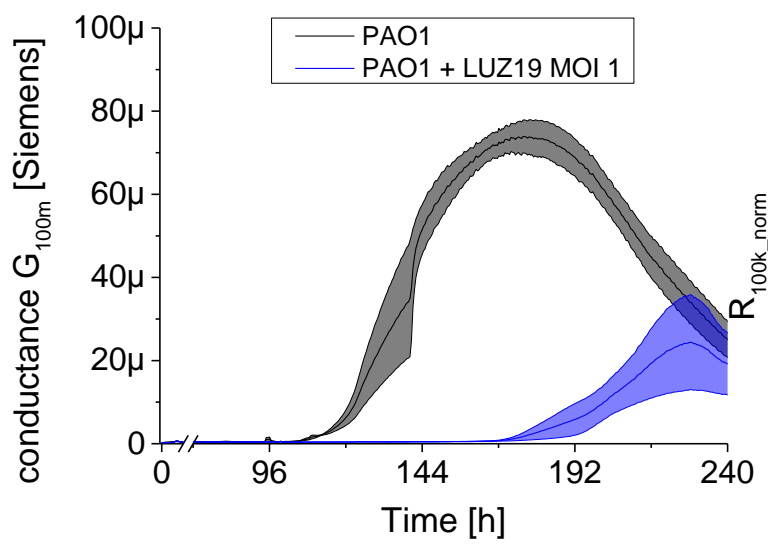

(e)

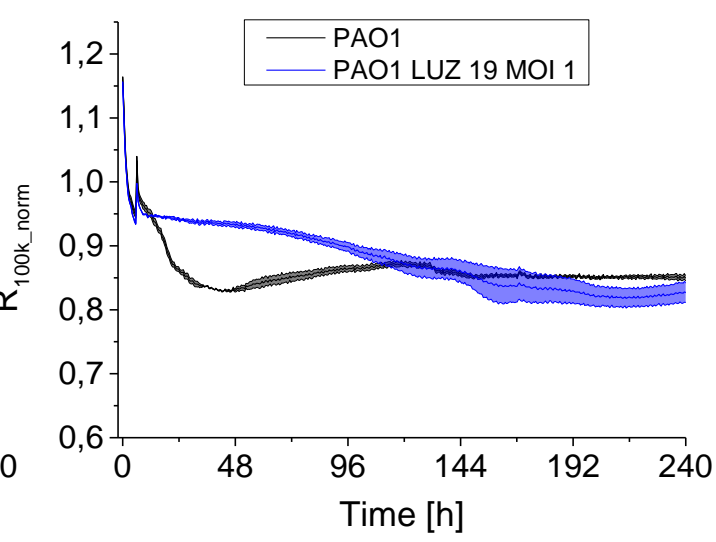

(b)

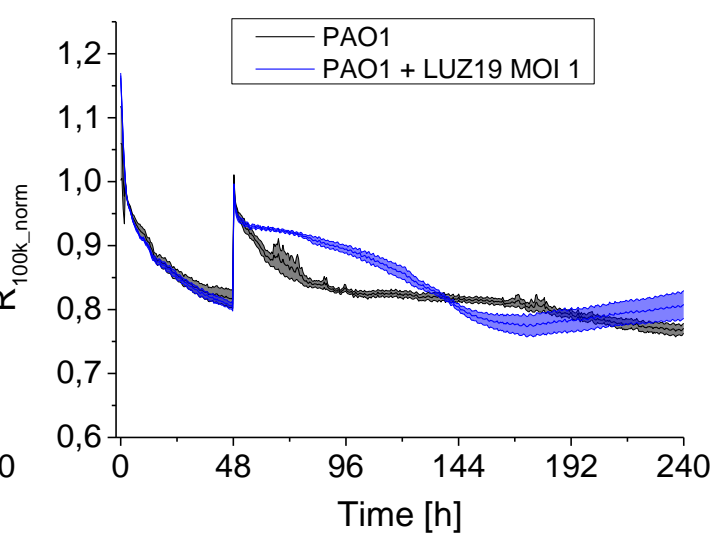

(d)

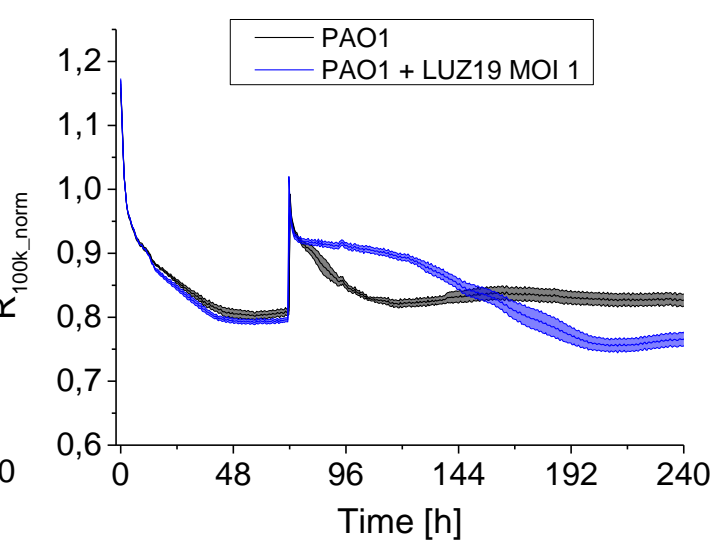

(f)

Figure 9. The changes in conductance G100m (left panel) and resistance R100k_norm (right panel) measured by QTF sensors during phage LUZ19 treatment of PAO1 biofilm at MOI = 1. Phage was applied at the 6 th $(\mathbf{a}, \mathbf{b}), 48$ th $(\mathbf{c}, \mathbf{d})$ and 72 nd $(\mathbf{e}, \mathbf{f})$ hour of biofilm culture. In the right panel, for each variant, a peak appears, which is associated with the transfer of QTF sensors to new medium containing an appropriate concentration of viral particles. Data are expressed as mean of conductance G100m \pm SD and resistance R100k_norm from three independent experiments. 


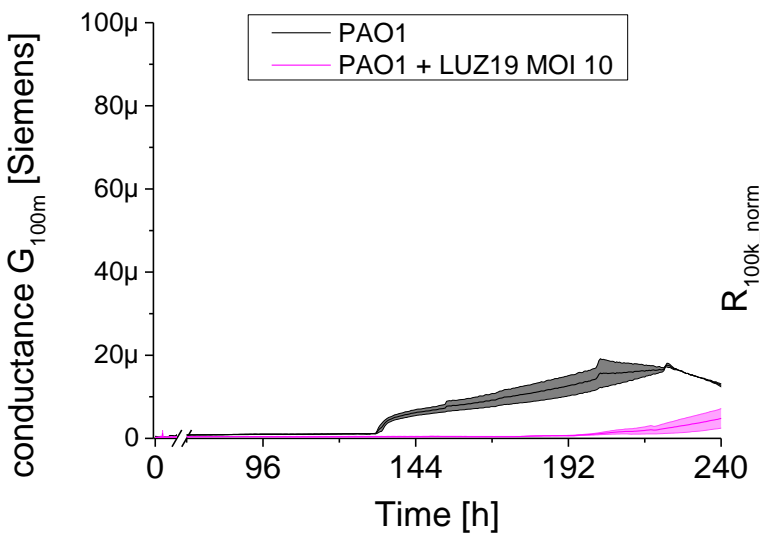

(a)

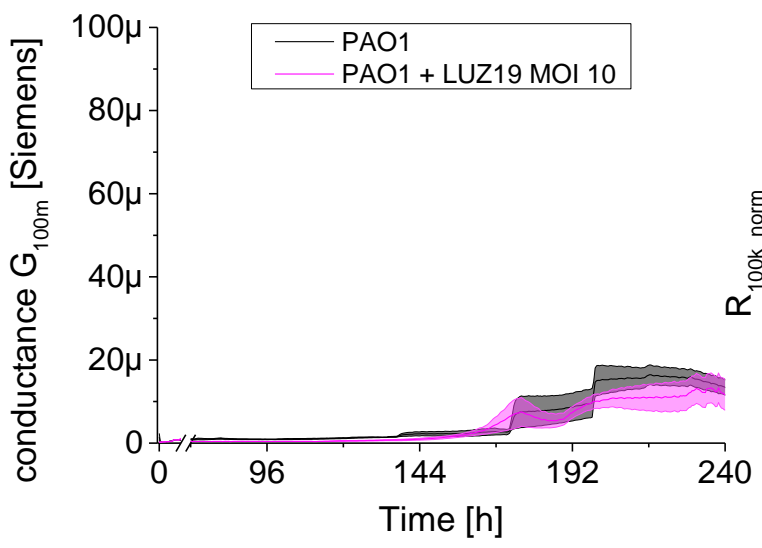

(c)

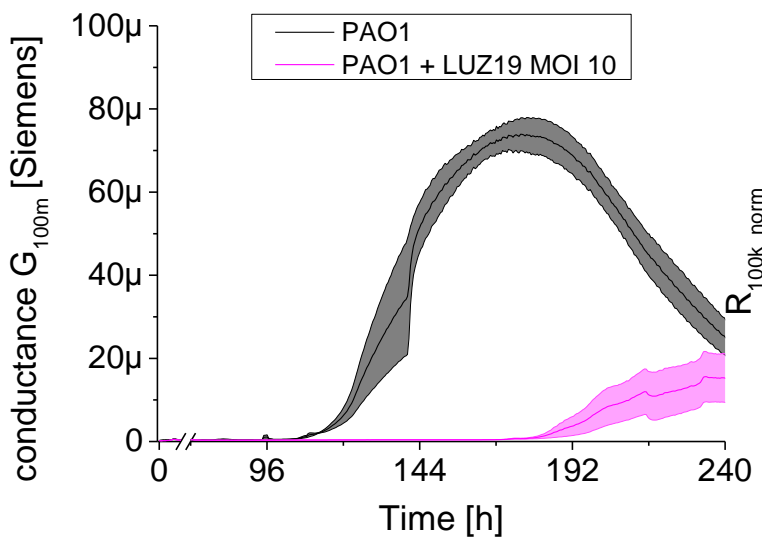

(e)

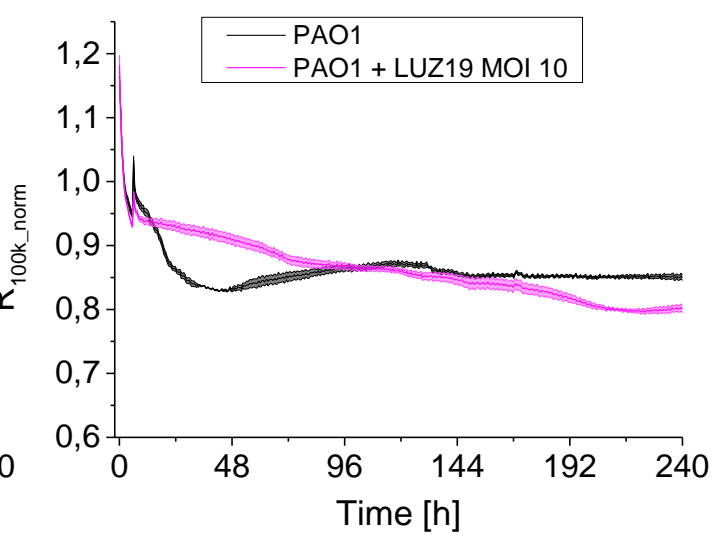

(b)

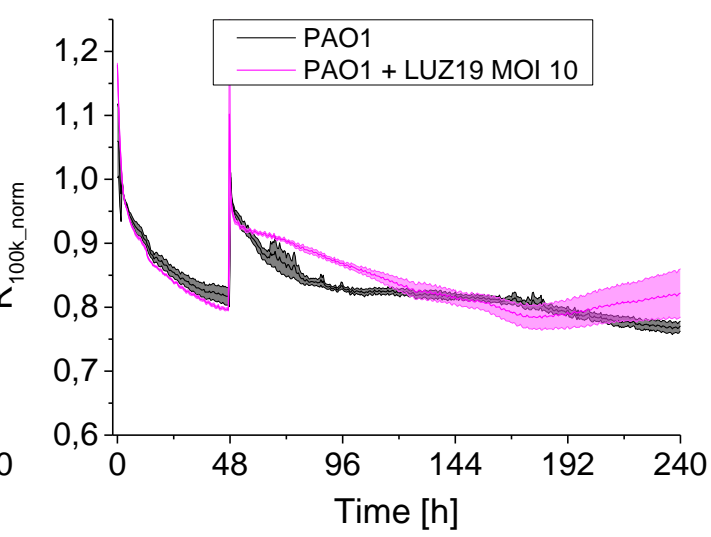

(d)

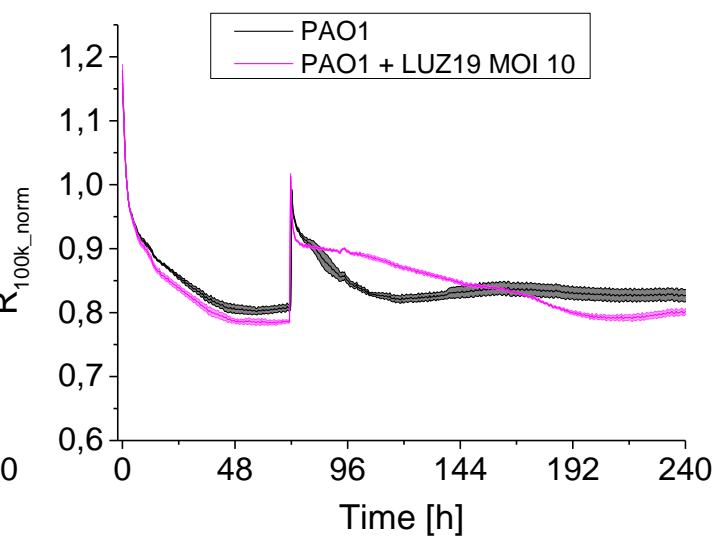

(f)

Figure 10. The changes in conductance $\mathrm{G}_{100 \mathrm{~m}}$ (left panel) and resistance $\mathrm{R}_{100 \mathrm{k} \_n o r m}$ (right panel) measured by QTF sensors during phage LUZ19 treatment of PAO1 biofilm at MOI = 10. Phage was applied at the 6th $(\mathbf{a}, \mathbf{b}), 48$ th $(\mathbf{c}, \mathbf{d})$ and 72 nd $(\mathbf{e}, \mathbf{f})$ hour of biofilm culture. In the right panel, for each variant, a peak appears, which is associated with the transfer of QTF sensors to new medium containing an appropriate concentration of viral particles. Data are expressed as mean of conductance $\mathrm{G}_{100 \mathrm{~m}} \pm \mathrm{SD}$ and resistance $R_{100 k \_n o r m}$ from three independent experiments. 


\section{Discussion}

Our previous experience with the application QTF sensor platform regarded biofilm biomass measurements when $P$. aeruginosa sessile cells were treated with antibiotics $[15,21]$. The changes in the resonant frequency of QTFs covered with biofilm structure were measured, which were then converted into the mass of bacterial biofilm at selected culture time points. That technology was limited to particular incubation times and it required complex material preparation procedures. In the presented study, we were looking for real-time measurement techniques remotely monitoring the biofilm formation and degradation. We have shown that the construction and arrangement of electrical covers of QTF allow for its application as an impedance spectroscopy sensor [18]. The applied impedance spectroscopy (IS) technique using QTF sensors is a convenient technology for biofilm growth monitoring in a real-time manner. IS was commonly used to determine the behavior of materials in chemical systems. It has become a tool for chemical and electrochemical analysis of materials with different levels of conductivity [21]. It has also found an application in the broadly understood biological sciences. Measurements of electrical parameters of biological objects have been monitored since around 1970, mainly to determine food contamination by bacteria [22]. Impedance spectroscopy is becoming widely used in experiments with bacterial biofilms as described by the Paredes group or van Duuren team [23,24]. Usually, these experiments base their measurements on a commercial instrument, the xCELLigence Real-Time Cell Analyzer (RTCA) from Acea Biosciences that measures impedance in 96 well plates equipped with gold microelectrodes by measuring the so-called cell index for Pseudomonas and Streptococcal biofilms [24,25]. Our previous experience also showed that IS using QTF is an effective technique for non-invasive analysis of biofilm culture parameters [18] extending now the aspect of the phage application model. The sensors used are planar and often the electrodes are finger-shaped. QTF sensor design is very convenient for spectra characterization and the creation of an electric equivalent model. In our technology, QTF sensors are immersed in bacterial culture enabling bacterial cells adhesion and an increase of biofilm monitoring. We also use a neutral metallization for tested objects, because QTF is mainly made of quartz and aluminum. The materials that make up the sensor are also relatively inexpensive, which is why the technology we propose is a relatively cheap tool for the monitoring of bacterial biofilm physiology.

The results obtained in our study are presented as simplified analyses measured in the form of conductance and resistance. Due to differences in the quality of QTF sensors, their surface functionalization in the culture medium and stabilization of temperature conditions (heating the TSB medium to $\left.37^{\circ} \mathrm{C}\right)$, the results for series resistance $\left(\mathrm{R}_{100 \mathrm{k}}\right)$ were normalized to three $\mathrm{h}$ of experiment and presented as $R_{100 k \_n o r m}$. Compared to commonly used methods, the undoubted advantage of IS based on QTF is the ability to measure parameters characterizing simultaneously the changes in planktonic forms in the culture medium and the biofilm formed on the sensor surface (parameters $\mathrm{R}_{100 \mathrm{k} \_n o r m}$ and $\mathrm{G}_{100 \mathrm{~m}}$, respectively). The dynamics of both parameters resulted from the equivalent electrical circuit (EEC) model we had previously set up and verified. The impedance spectroscopy measurements are highly repeatable at a high resolution. The spectra collection took place for the same sensor every $30 \mathrm{~min}$. This allowed us to obtain reliable results, without the need for complex sample manipulation, as is the case with many recommended biofilm monitoring methods that have so far been described in Crit Rev Microbiol [13].

Most of the laboratory methods used to determine biofilm growth rely on the measurements at a given culture point. This applies to methods based on CFU/mL calculation, as well as staining techniques [26]. Moreover, these experiments are usually limited to several hours of incubation, and in many cases, we are not able to characterize the phenomenon of biofilm dynamic changes. In the case of IS using QTF, the experiments were carried out after up to 10 days of incubation. Our measurements were carried out semi-parallel to each sensor. The built platform allowed 24 independent experiments to be performed simultaneously, while the entire measurement took place in a titration plate in batch culture.

We observed a clear relationship between the series resistance parameter $\left(\mathrm{R}_{100 \mathrm{k} \_ \text {norm }}\right)$ and conductance $\left(G_{100 m}\right)$. When $R_{100 k \_n o r m}$ resistance reached the plateau phase, $G_{100 m}$ conductance increased significantly. 
The lowering resistance is associated with an increase in planktonic cell number in the medium (described earlier in [18]). The increase in the conductance is identified with the appearance and level of surface coverage by a conductive EPS matrix. Based on the $\mathrm{G}_{100 \mathrm{~m}}$ parameter, we measured the QTF coverage with an EPS matrix that changes the charge transfer across the barrier between the electrode and electrolyte. However, the drop in the conductance parameter for $\mathrm{G}_{100 \mathrm{~m}}$ is associated with a reduction of EPS or a change in the physicochemical properties of the biofilm matrix. The culture was carried out in stationary conditions, without medium exchange, therefore, the conductance reduction scenario was associated with the reuse of matrix components as an energy source of bacterial culture. The SEM visualization of QTF surface on the 6 th, 8 th and 10th day of biofilm formation combined with the colony count results provide the proof that the above conclusions were correct.

We used a QTF as an impedance sensor to measure electrical changes of PAO1 culture during phage LUZ19 infection at three different MOIs corresponding to $0.1,1$ and 10. An inhibitory effect on biofilm formation was seen when the infection took place at the 6th and $72 \mathrm{nd} \mathrm{h}$ of incubation, regardless of MOI applied. It has also been shown that generally, the biofilm dynamics were independent of phage LUZ19 infecting dose. Interestingly, 48-h biofilm turned out to be a breakthrough moment, because at this time phage administration did not affect the biofilm formation, although the killing influence on both sessile and planktonic cells were recorded. Comparing the results from three different biofilm monitoring techniques (colony count, SEM, and impedance), we may conclude that G100m parameter shows, in fact, the amount of the biofilm EPS accumulating in the sensor surface.

The impedance spectroscopy model we designed is able to monitor the behavior of planktonic culture surrounding the QTF sensor. The $\mathrm{R}_{100 \mathrm{k} \_n o r m}$ parameter indicating the culture propagation dynamics in the case of phage infection means the inhibition of phage-sensitive cell growth and secondary regrowth events of selected phage resistant bacteria. That population regrowth and dynamic phage-bacteria balance in the biofilm structure was confirmed in the colony count detection.

The application of Pseudomonas phages in order to eradicate bacterial biofilm is widely described in the literature showing both successes and failures. Some phages like phage E27 or M4, are not able to penetrate the biofilm matrix, thus, they are ineffective in biofilm elimination $[27,28]$. The strong restriction of phage access to cells embedded in the dense biofilm EPS matrix can be partially overcome by virion-associated depolymerases produced by some phages such as LUZ19, tested in the presented study [29]. Phage infection usually led to a significant cell number reduction ( 3 logs) on catheter surfaces

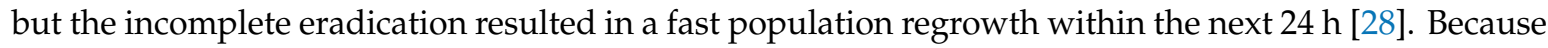
of the presence of phage insensitive portions in the sessile cells, the idea of combined phage-antibiotic treatment was also studied [30]. The synergy effect of podovirus phage LUZ7 and streptomycin was noticed against $P$. aeruginosa PAO1 biofilm compared to a single preparation [30]. A similar synergy observation was done by the Knezevic group [31]. Nevertheless, the heterogenic character of the sessile population protects the biofilm from a complete elimination regardless of an applied anti-biofilm agent. The limitation in the biofilm eradication is also caused by rapid emergence of phage-resistant phenotypes [32,33].

As phages are co-evolving with its host, there are some contrary mechanisms of phage influence on biofilm formation and maintenance. This specifically concerns prophages having a huge impact on bacterial phenotypes. It is already proven that the remodeling of a biofilm EPS matrix and the development of small-colony phenotypic variants are associated with the presence of filamentous prophage Pf4 (Pf) in P. aeruginosa genome [34]. It turns out that Pf participates in the organization of biofilm matrix, while being induced, release and transform into crystal lattices. This modification of matrix improves the adhesion process and increases drying and antibiotics tolerance [34]. Tobramycin applied with the biofilm treatment accumulates inside liquid crystals of Pf phage particles, whereas a strong packaging of negatively polarized phage polymers interact with positively charged antibiotics [35]. Pf phage activity is not only limited to the modification of a specific host EPS matrix, but may affect the biofilm growth of distant accompanying organisms (Aspergillus fumigatus), by direct capture of $\mathrm{Fe}^{3+}$ ions (and other multivalent metals) [36,37]. Above 
prophage activity is a newly discovered mechanism of restrictive regulation of the biofilms especially in $P$. aeruginosa strains associated with cystic fibrosis patient infection.

In conclusion, the technology we presented in this study gives a reproducible and precise measurement of biofilm structure and planktonic culture dynamics analyzed simultaneously and in a real-time manner without specific sample preparation. The impedance parameters such as the conductance and resistance of biological samples give more insight into the bacterial culture changes happening during the natural growth and the fluctuation of culture characteristics when treated with antibacterial agents. Moreover, the impedance parameters turned out to be specific for EPS amount measurement. Therefore this system seems to be very useful in the monitoring of antibacterial effects in biofilm prevention and eradication, and especially for the study on phage influence on biofilm matrix development.

Author Contributions: Conceptualization, G.G. and Z.D.-K.; methodology, G.G. and T.P.; software, T.P. and P.S.; validation and investigation, T.P., P.S., S.G., G.G.; resources and funding, Z.D.-K. and T.G.; data curation, G.G., T.P. and Z.D.-K.; manuscript preparation G.G., T.P. and Z.D.-K. All authors have read and agreed to the published version of the manuscript.

Funding: This study was partially supported by research grant UMO-2016/21/B/NZ6/01157 of National Science Centre, Poland.

Acknowledgments: We would like to thank Marek Chmielewski, for his valuable help in biofilm visualization by SEM microscopy.

Conflicts of Interest: The authors declare no conflict of interest.

\section{Abbreviations}

$\begin{array}{ll}\text { QTF } & \text { Quartz Tuning Fork } \\ \mathbf{G}_{\mathbf{1 0 0 m}} & \text { conductance of simple analysis measured at frequency } 100 \mathrm{mHz} \\ \mathbf{R}_{\mathbf{1 0 0 k} \text { norm }} & \text { normalized resistance of simple analysis measured at frequency } 100 \mathrm{kHz} \\ \text { EPS } & \text { extracellular polymeric substances } \\ \text { IS } & \text { impedance spectroscopy } \\ \mathbf{Z} & \text { impedance module } \\ \text { G } & \text { conductance } \\ \text { TSB } & \text { tryptic soy broth }\end{array}$

\section{References}

1. Costerton, J.W. Introduction to biofilm. Int. J. Antimicrob. Agents 1999, 11, 217-221. [CrossRef]

2. Hall-Stoodley, L.; Costerton, J.W.; Stoodley, P. Bacterial biofilms: From the natural environment to infectious diseases. Nat. Rev. Microbiol. 2004, 2, 95-108. [CrossRef] [PubMed]

3. Olszak, T.; Shneider, M.M.; Latka, A.; Maciejewska, B.; Browning, C.; Sycheva, L.V.; Cornelissen, A.; Danis-Wlodarczyk, K.; Senchenkova, S.N.; Shashkov, A.S.; et al. The O-specific polysaccharide lyase from the phage LKA1 tailspike reduces Pseudomonas virulence. Sci. Rep. 2017, 7. [CrossRef]

4. Burrowes, B.; Harper, D.R.; Anderson, J.; McConville, M.; Enright, M.C. Bacteriophage therapy: Potential uses in the control of antibiotic-resistant pathogens. Expert Rev. Anti-Infect. Ther. 2011, 9, 775-785. [CrossRef] [PubMed]

5. Percival, S.L.; Hill, K.E.; Williams, D.W.; Hooper, S.J.; Thomas, D.W.; Costerton, J.W. A review of the scientific evidence for biofilms in wounds. Wound Repair Regen. 2012, 20, 647-657. [CrossRef] [PubMed]

6. Chibani-Chennoufi, S.; Bruttin, A.; Dillmann, M.L.; Brüssow, H. Phage-host interaction: An ecological perspective. J. Bacteriol. 2004, 186, 3677-3686. [CrossRef]

7. Olszak, T.; Danis-Wlodarczyk, K.; Arabski, M.; Gula, G.; Maciejewska, B.; Wasik, S.; Lood, C.; Higgins, G.; Harvey, B.J.; Lavigne, R.; et al. Pseudomonas aeruginosa PA5oct Jumbo Phage Impacts Planktonic and Biofilm Population and Reduces Its Host Virulence. Viruses 2019, 11, 1089. [CrossRef]

8. O'Toole, G.A.; Kolter, R. Flagellar and twitching motility are necessary for Pseudomonas aeruginosa biofilm development. Mol. Microbiol. 1998, 30, 295-304. [CrossRef] 
9. Boe-Hansen, R.; Martiny, A.C.; Arvin, E.; Albrechtsen, H.J. Monitoring biofilm formation and activity in drinking water distribution networks under oligotrophic conditions. Water Sci. Technol. 2003, 47, 91-97. [CrossRef]

10. Chen, M.Y.; Lee, D.J.; Tay, J.H.; Show, K.Y. Staining of extracellular polymeric substances and cells in bioaggregates. Appl. Microbiol. Biotechnol. 2007, 75, 467-474. [CrossRef]

11. Zhang, T.; Fang, H. Distribution of extracellular polysaccharides in the anaerobic granular sludges. Environ. Biotechnol. Adv. Water Wastewater Appl. Trop. Sel. Proc. Iwa Int. Conf. Environ. Biotechnol. 2004, 153-158.

12. Chen, M.Y.; Lee, D.J.; Tay, J.H. Extracellular polymeric substances in fouling layer. Sep. Sci. Technol. 2006, 41, 1467-1474. [CrossRef]

13. Azeredo, J.; Azevedo, N.F.; Briandet, R.; Cerca, N.; Coenye, T.; Costa, A.R.; Desvaux, M.; Di Bonaventura, G.; Hébraud, M.; Jaglic, Z.; et al. Critical review on biofilm methods. Crit. Rev. Microbiol. 2017, 43, 313-351. [CrossRef] [PubMed]

14. Hannig, C.; Follo, M.; Hellwig, E.; Al-Ahmad, A. Visualization of adherent micro-organisms using different techniques. J. Med. Microbiol. 2010, 59, 1-7. [CrossRef]

15. Waszczuk, K.; Gula, G.; Swiatkowski, M.; Olszewski, J.; Herwich, W.; Drulis-Kawa, Z.; Gutowicz, J.; Gotszalk, T. Evaluation of Pseudomonas aeruginosa biofilm formation using piezoelectric tuning fork mass sensors. Sens. Actuators B Chem. 2012, 170, 7-12. [CrossRef]

16. Danis-Wlodarczyk, K.; Olszak, T.; Arabski, M.; Wasik, S.; Majkowska-Skrobek, G.; Augustyniak, D.; Gula, G.; Briers, Y.; Jang, H.B.; Vandenheuvel, D.; et al. Characterization of the newly isolated lytic bacteriophages KTN6 and KT28 and their efficacy against Pseudomonas aeruginosa biofilm. PLoS ONE 2015, 10. [CrossRef]

17. Danis-Wlodarczyk, K.; Vandenheuvel, D.; Jang, H.B.; Briers, Y.; Olszak, T.; Arabski, M.; Wasik, S.; Drabik, M.; Higgins, G.; Tyrrell, J.; et al. A proposed integrated approach for the preclinical evaluation of phage therapy in Pseudomonas infections. Sci. Rep. 2016, 6. [CrossRef]

18. Piasecki, T.; Guła, G.; Nitsch, K.; Waszczuk, K.; Drulis-Kawa, Z.; Gotszalk, T. Evaluation of Pseudomonas aeruginosa biofilm formation using Quartz Tuning Forks as impedance sensors. Sens. Actuators B Chem. 2013, 189, 60-65. [CrossRef]

19. Piasecki, T.; Chabowski, K.; Nitsch, K. Design, calibration and tests of versatile low frequency impedance analyser based on ARM microcontroller. Measurement 2016, 91, 155-161. [CrossRef]

20. Barsoukov, E.; Macdonald, J.R. Impedance Spectroscopy. In Theory, Experiment and Applications, 2nd ed.; Wiley Interscience: New York, NY, USA, 2005.

21. Lai, W.; Haile, S.M. Impedance Spectroscopy as a Tool for Chemical and Electrochemical Analysis of Mixed Conductors: A Case Study of Ceria. J. Am. Ceram. Soc. 2005, 88, 2979-2997. [CrossRef]

22. Cady, P. Rapid automated bacterial identification by impedance measurement. In New Approaches to the Identification of Microorgan-isms; Heden, C.G., Ed.; John Wiley: New York, NY, USA, 1975; pp. 73-99.

23. Paredes, J.; Becerro, S.; Arizti, F.; Aguinaga, A.; Del Pozo, J.L.; Arana, S. Interdigitated microelectrode biosensor for bacterial biofilm growth monitoring by impedance spectroscopy technique in 96 -well microtiter plates. Sens. Actuators B Chem. 2013, 178, 663-670. [CrossRef]

24. Van Duuren, J.B.J.H.; Müsken, M.; Karge, B.; Tomasch, J.; Wittmann, C.; Häussler, S.; Brönstrup, M. Use of Single-Frequency Impedance Spectroscopy to Characterize the Growth Dynamics of Biofilm Formation in Pseudomonas aeruginosa. Sci. Rep. 2017, 7. [CrossRef] [PubMed]

25. Gutiérrez, D.; Hidalgo-Cantabrana, C.; Rodríguez, A.; García, P.; Ruas-Madiedo, P. Monitoring in Real Time the Formation and Removal of Biofilms from Clinical Related Pathogens Using an Impedance-Based Technology. PLoS ONE 2016, 11, e0163966. [CrossRef] [PubMed]

26. Knezevic, P.; Petrovic, O. A colorimetric microtiter plate method for assessment of phage effect on Pseudomonas aeruginosa biofilm. J. Microbiol. Methods 2008, 74, 114-118. [CrossRef] [PubMed]

27. Doolittle, M.M.; Cooney, J.J.; Caldwell, D.E. Tracing the interaction of bacteriophage with bacterial biofilms using fluorescent and chromogenic probes. J. Ind. Microbiol. 1996, 16, 331-341. [CrossRef]

28. Fu, W.; Forster, T.; Mayer, O.; Curtin, J.J.; Lehman, S.M.; Donlan, R.M. Bacteriophage cocktail for the prevention of biofilm formation by Pseudomonas aeruginosa on catheters in an in vitro model system. Antimicrob. Agents Chemother. 2010, 54, 397-404. [CrossRef]

29. Latka, A.; Maciejewska, B.; Majkowska-Skrobek, G.; Briers, Y.; Drulis-Kawa, Z. Bacteriophage-encoded virion-associated enzymes to overcome the carbohydrate barriers during the infection process. Appl. Microbiol. Biotechnol. 2017, 101, 3103-3119. [CrossRef] 
30. Torres-Barceló, C.; Arias-Sánchez, F.I.; Vasse, M.; Ramsayer, J.; Kaltz, O.; Hochberg, M.E. A Window of Opportunity to Control the Bacterial Pathogen Pseudomonas aeruginosa Combining Antibiotics and Phages. PLoS ONE 2014, 9, e106628. [CrossRef]

31. Knezevic, P.; Curcin, S.; Aleksic, V.; Petrusic, M.; Vlaski, L. Phage-antibiotic synergism: A possible approach to combatting Pseudomonas aeruginosa. Res. Microbiol. 2013, 164, 55-60. [CrossRef]

32. Pires, D.; Sillankorva, S.; Faustino, A.; Azeredo, J. Use of newly isolated phages for control of Pseudomonas aeruginosa PAO1 and ATCC 10145 biofilms. Res. Microbiol. 2011, 162, 798-806. [CrossRef]

33. Webb, J.S.; Lau, M.; Kjelleberg, S. Bacteriophage and phenotypic variation in Pseudomonas aeruginosa biofilm development. J. Bacteriol. 2004, 186, 8066-8073. [CrossRef] [PubMed]

34. Secor, P.R.; Sweere, J.M.; Michaels, L.A.; Malkovskiy, A.V.; Lazzareschi, D.; Katznelson, E.; Rajadas, J.; Birnbaum, M.E.; Arrigoni, A.; Braun, K.R.; et al. Filamentous bacteriophage promote biofilm assembly and function. Cell Host Microbe 2015, 18, 549-559. [CrossRef] [PubMed]

35. Wnorowska, U.; Niemirowicz, K.; Myint, M.; Diamond, S.L.; Wróblewska, M.; Savage, P.B.; Janmey, P.A.; Bucki, R. Bactericidal activities of cathelicidin LL-37 and select cationic lipids against the hypervirulent Pseudomonas aeruginosa strain LESB58. Antimicrob. Agents Chemother. 2015, 59, 3808-3815. [CrossRef] [PubMed]

36. Nazik, H.; Penner, J.C.; Ferreira, J.A.; Haagensen, J.A.J.; Cohen, K.; Spormann, A.M.; Martinez, M.; Chen, V.; Hsu, J.L.; Clemons, K.V.; et al. Effects of iron chelators on the formation and development of Aspergillus fumigatus biofilm. Antimicrob. Agents Chemother. 2015, 59, 6514-6520. [CrossRef]

37. Penner, J.C.; Ferreira, J.A.G.; Secor, P.R.; Sweere, J.M.; Birukova, M.K.; Joubert, L.M.; Haagensen, J.A.J.; Garcia, O.; Malkovskiy, A.V.; Kaber, G.; et al. Pf4 bacteriophage produced by Pseudomonas aeruginosa inhibits Aspergillus fumigatus metabolism via iron sequestration. Microbiology (United Kingdom) 2016, 162, 1583-1594. [CrossRef]

(C) 2020 by the authors. Licensee MDPI, Basel, Switzerland. This article is an open access article distributed under the terms and conditions of the Creative Commons Attribution (CC BY) license (http://creativecommons.org/licenses/by/4.0/). 